\title{
Gaps induced by inversion symmetry breaking and second-generation Dirac cones in graphene/hexagonal boron nitride
}

\author{
Eryin Wang ${ }^{1 \dagger}$, Xiaobo Lu ${ }^{2 \dagger}$, Shijie Ding ${ }^{1}$, Wei Yao ${ }^{1}$, Mingzhe Yan ${ }^{1}$, Guoliang Wan ${ }^{1}$, Ke Deng ${ }^{1}$, \\ Shuopei Wang ${ }^{2}$, Guorui Chen ${ }^{3}$, Liguo Ma ${ }^{3}$, Jeil Jung ${ }^{4}$, Alexei V. Fedorov ${ }^{5}$, Yuanbo Zhang ${ }^{3}$, \\ Guangyu Zhang ${ }^{2,6}$ and Shuyun Zhou ${ }^{1,6 \star}$
}

\begin{abstract}
Graphene/hexagonal boron nitride (h-BN) has emerged as a model van der Waals heterostructure ${ }^{1}$ as the superlattice potential, which is induced by lattice mismatch and crystal orientation, gives rise to various novel quantum phenomena, such as the self-similar Hofstadter butterfly states ${ }^{2-5}$. Although the newly generated second-generation Dirac cones (SDCs) are believed to be crucial for understanding such intriguing phenomena, fundamental knowledge of SDCs, such as locations and dispersion, and the effect of inversion symmetry breaking on the gap opening, still remains highly debated due to the lack of direct experimental results. Here we report direct experimental results on the dispersion of SDCs in $0^{\circ}$. aligned graphene/h-BN heterostructures using angle-resolved photoemission spectroscopy. Our data unambiguously reveal SDCs at the corners of the superlattice Brillouin zone, and at only one of the two superlattice valleys. Moreover, gaps of approximately $100 \mathrm{meV}$ and approximately $160 \mathrm{meV}$ are observed at the SDCs and the original graphene Dirac cone, respectively. Our work highlights the important role of a strong inversion-symmetry-breaking perturbation potential in the physics of graphene/h-BN, and fills critical knowledge gaps in the band structure engineering of Dirac fermions by a superlattice potential.
\end{abstract}

Hexagonal boron nitride (h-BN) shares a similar honeycomb lattice structure to graphene, yet its lattice is stretched by $1.8 \%$. Moreover, the breaking of the inversion symmetry by distinct boron and nitrogen sublattices leads to a large bandgap $(5.97 \mathrm{eV})$ in the $\pi$ band, which is in sharp contrast to the gapless Dirac cones in graphene. By stacking graphene atop h-BN to form a van der Waals heterostructure ${ }^{1}$, graphene/h-BN not only exhibits greatly improved properties for device applications, such as reduced ripples, suppressed charge inhomogeneities and higher mobility ${ }^{6,7}$, but also provides unique opportunities for band structure engineering of Dirac fermions by a periodic potential ${ }^{8,9}$. The superlattice potential induced by the lattice mismatch and crystal orientation can significantly modify the electronic properties of graphene and lead to various novel quantum phenomena, for example, the emergence of second-generation Dirac cones (SDCs), which are crucial for the realization of Hofstadter butterfly states under an applied magnetic field ${ }^{2-5}$, renormalization of the Fermi velocity ${ }^{8,10-12}$, gap opening at the Dirac point $t^{4,13-16}$, topological currents ${ }^{15}$ and gate-dependent pseudospin mixing ${ }^{17}$. Hence, understanding the effects of the superlattice potential on the band structure of graphene is crucial for advancing its device applications, and for gaining new knowledge about the fundamental physics of Dirac fermions in a periodic potential.

Previously, the existence of SDCs has been deduced from scanning tunnelling spectroscopy, resistivity and capacitance measurements ${ }^{2,5,18,19}$. However, such measurements are not capable of mapping out the electronic dispersion with momentum-resolved information, and the lack of direct experimental results has led to ambiguous and even conflicting results about the electronic spectra of SDCs and the existence of gaps. Although various theoretical models have been proposed ${ }^{10,11,20-23}$, the locations and dispersions of SDCs depend strongly on the parameters used to describe the inversion-symmetric and inversion-asymmetric superlattice potential modulation ${ }^{10}$. Different choices of inversion-symmetric perturbation could result in either isolated or overlapping $\mathrm{SDCs}^{10}$, and the locations of SDCs could change from the edges of the superlattice Brillouin zone $(\mathrm{SBZ})^{9,10}$ to the corners ${ }^{10,11,21}$. The inversionasymmetric perturbation potential can strongly affect the gap opening at the Dirac point. The gap opening in graphene/h-BN is a highly debated issue, with some theoretical and experimental studies arguing for its existence $e^{4,12,14,19,21,23-25}$ whereas others rule it out ${ }^{2,6-8,11}$. Such knowledge gaps in understanding the fundamental electronic structure of graphene/h-BN call for a careful examination of the electronic structure by angle-resolved photoemission spectroscopy (ARPES), which can map out the dispersions of the original Dirac cone and the SDCs with both energy- and momentum-resolution and detect the gap opening directly if there is any.

ARPES studies of graphene/h-BN heterostructures have been challenging for several reasons. First, the size of the heterostructure prepared by transferring graphene atop the h-BN substrate was typically a few micrometres $(\mu \mathrm{m})$, much smaller than the typical ARPES beam size of $50-100 \mu \mathrm{m}$. Second, because of the large moiré periodicity $(\sim 14 \mathrm{~nm}, 56$ times the lattice constant of graphene for $0^{\circ}$-aligned graphene/h-BN), the separation between the original Dirac cone of graphene and the cloned Dirac cones is extremely small, on the order of the reciprocal superlattice vector $G_{s} \approx 0.05 \AA^{-1}$. Resolving band dispersions

\footnotetext{
${ }^{1}$ State Key Laboratory of Low Dimensional Quantum Physics and Department of Physics, Tsinghua University, Beijing 100084, China. ${ }^{2}$ Beijing National Laboratory for Condensed Matter Physics and Institute of Physics, Chinese Academy of Sciences, Beijing 100190, China. ${ }^{3}$ State Key Laboratory of Surface Physics and Department of Physics, Fudan University, Shanghai 200433, China. ${ }^{4}$ Department of Physics, University of Seoul, Seoul 02504, Korea. ${ }^{5}$ Advanced Light Source, Lawrence Berkeley National Laboratory, Berkeley, California 94720, USA. ${ }^{6}$ Collaborative Innovation Center of Quantum Matter, Beijing, China. ${ }^{\dagger}$ These authors contributed equally to this work. ${ }^{\star}$ e-mail: syzhou@mail.tsinghua.edu.cn
} 
a

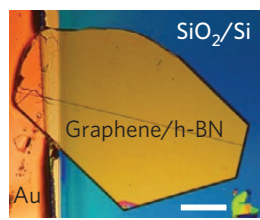

b

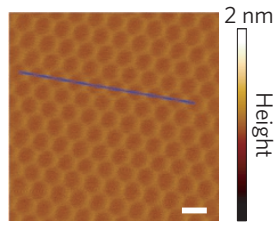

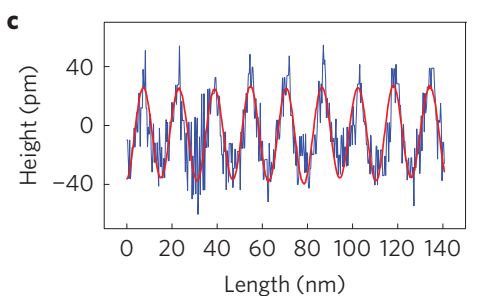

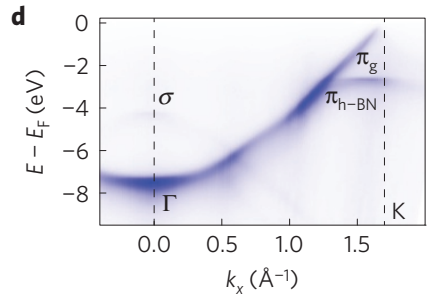

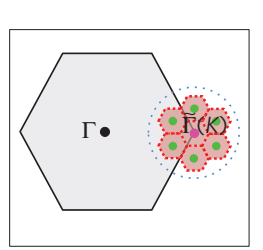

f

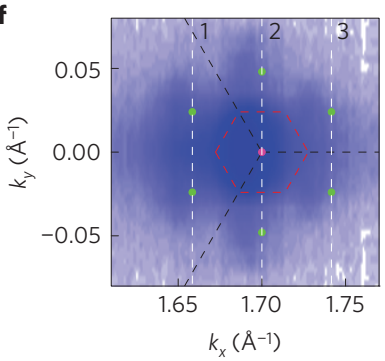

g

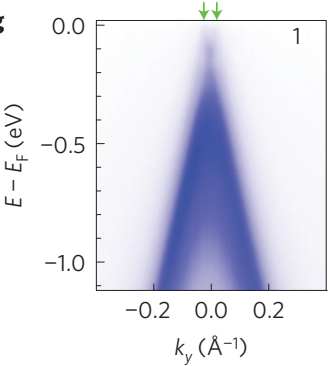

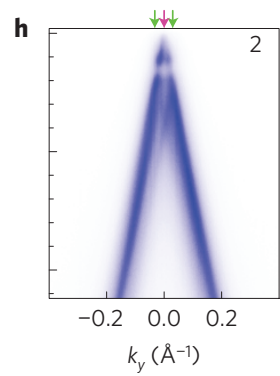

Figure 1 | Six cloned first-generation Dirac cones from the superlattice potential. a, Optical micrograph image of a typical sample measured. The white scale bar is $100 \mu \mathrm{m}$. b. Raw image of the moiré pattern formed in graphene/h-BN from atomic force microscopy taken in tapping mode in the ambient atmosphere. The white scale bar is $20 \mathrm{~nm}$. c. Height profile along the blue line in $\mathbf{b}$. The raw data and the data after high-pass-filtered inverse fast Fourier transformation are shown in blue and red, respectively. $\mathbf{d}$, Dispersion along the $\Gamma-\mathrm{K}$ direction showing the $\sigma$ and $\pi$ bands from graphene and h-BN. e, Schematic drawing of the Brillouin zones of graphene (grey hexagon) and the SBZs (red dotted hexagons). The $\Gamma$ (black circle), $\tilde{\Gamma}$ (K) (pink circle) points of the graphene Brillouin zone and the six nearest SBZ centres (green circles) are shown. $\mathbf{f}$, Constant-energy map at $E_{F}$. Replicas are observed around the six nearest SBZ centres (green circles). The Brillouin zones of graphene and the superlattice are indicated by black and red broken lines, respectively. $\mathbf{g}-\mathbf{i}$, Dispersions along cuts 1, 2 and 3 indicated in $\mathbf{f}$. The tops of the original Dirac cone and the replicas are indicated by pink and green arrows, respectively.

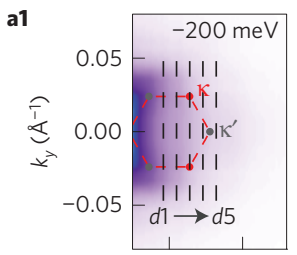

b1
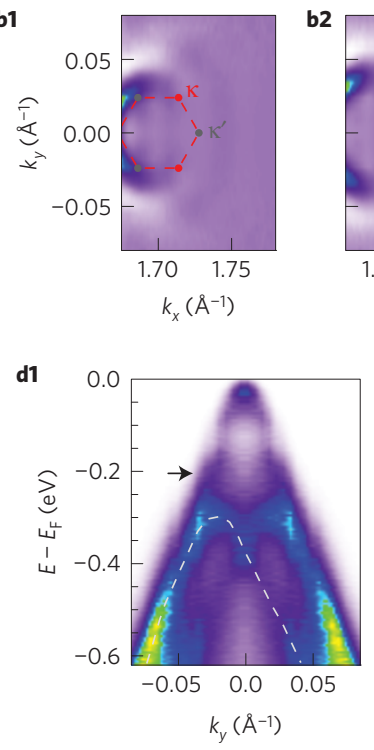

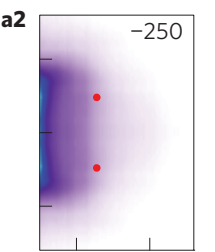

b2

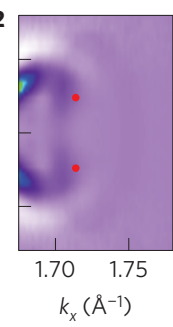

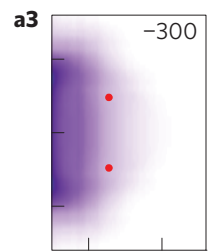

b3

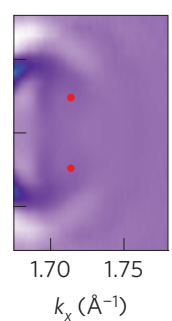

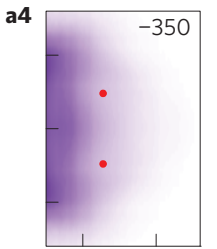

b4

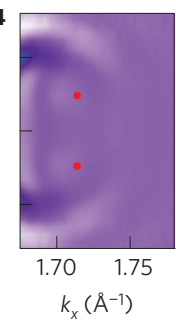

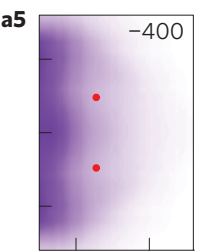

b5

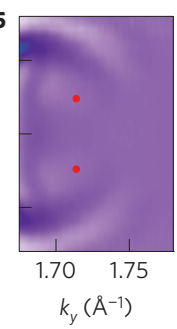

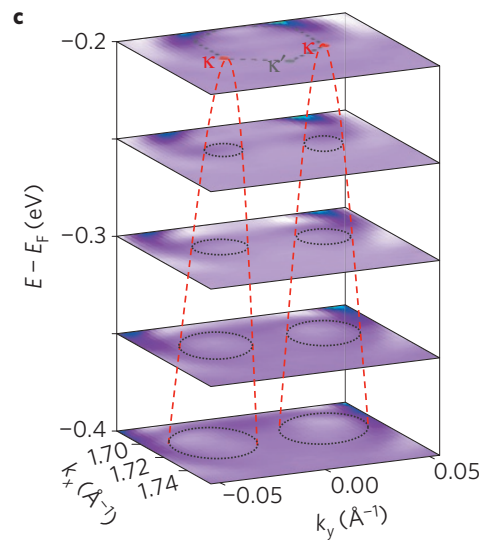

d2

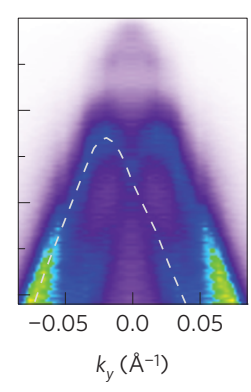

d3

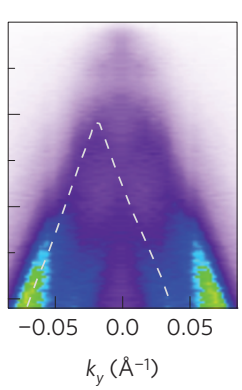

d4

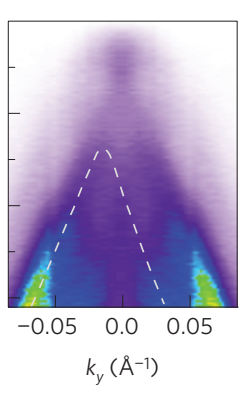

d5

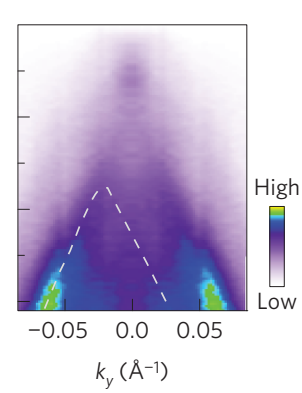

Figure 2 | Emergence of second-generation Dirac cones. a1-a5, Constant-energy maps at $-200,-250,-300,-350$ and -400 meV.

b1-b5, Constant-energy maps of EDC curvature at $-200,-250,-300,-350$ and $-400 \mathrm{meV}$. The moiré SBZ is shown as a broken red line. Red and grey dots indicate the two inequivalent sets of SBZ corners, $\kappa$ and $\kappa^{\prime}$. c . Stacking of constant-energy maps of EDC curvature to show the conical dispersion at the two $\kappa$ points. d1-d5, Dispersions along cuts d1-d5 shown in a1. The white dashed lines are guides for the evolution of SDCs dispersions. The black arrow indicates the dispersion from the original Dirac cone.

within such a small momentum space requires samples of extremely high quality with sharp spectral features. Recently, highquality $0^{\circ}$-aligned graphene/h-BN heterostructures with large sizes extending a few hundred micrometres have been successfully grown directly by remote plasma-enhanced chemical vapour deposition $(\mathrm{R}-\mathrm{PECVD})^{19}$; this has made our ARPES studies possible. 

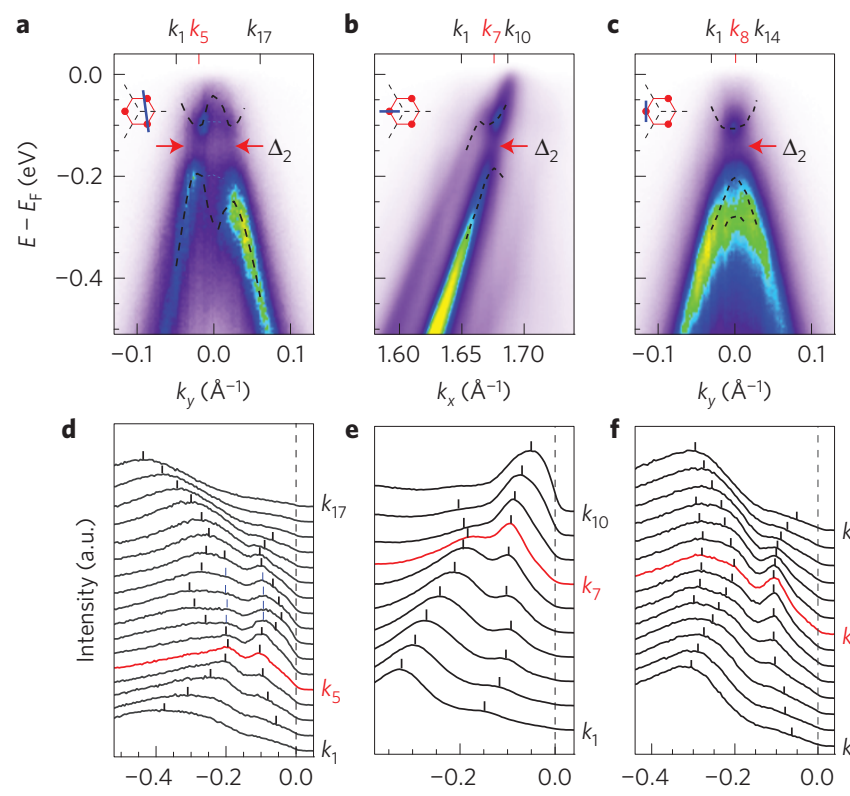

$k_{x}\left(\AA^{-1}\right)$

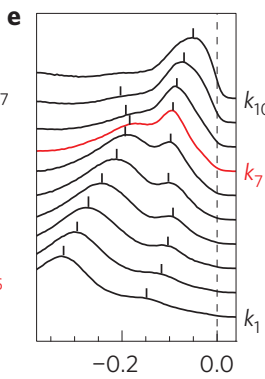

$$
k_{y}\left(\AA^{-1}\right)
$$

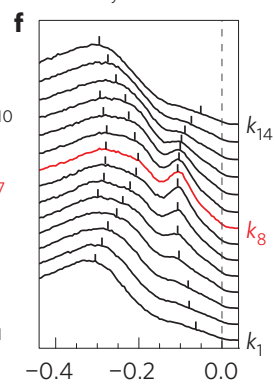

$E-E_{\mathrm{F}}(\mathrm{eV})$

g
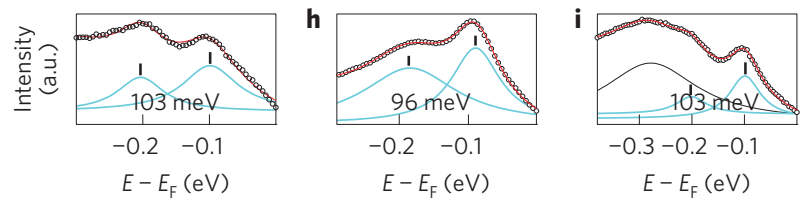

Figure 3 | Observation of gap opening at second-generation Dirac points. a-c, ARPES data through the SDPs along different directions. Black dashed lines are guides for the dispersion extracted from EDCs. Blue dotted lines indicate the non-dispersive states from impurity scattering. The insets are schematic drawings of the measurement geometry. The graphene and superlattice Brillouin zones are indicated by black dashed and red solid lines, respectively. The red dots represent the $\kappa$ points of the SBZ. $\mathbf{d - f}$, EDCs between the momenta indicated in a-c. The EDCs across the SDPs are highlighted by red lines. $\mathbf{g}-\mathbf{i}$, Fitting results of the EDCs across the SDPs in a-c with two $(\mathbf{g}, \mathbf{h})$ or three (i) Lorentzian peaks. The energy separation between the fitted Lorentzian peaks (ticks on cyan curves) indicates the gap size.

Figure 1a,b shows optical and atomic force microscopy images, respectively, of a typical graphene/h-BN sample that we have measured ${ }^{19}$. A palladium (Pd) or gold $(\mathrm{Au})$ electrode was deposited to ground the sample to avoid charging during ARPES measurements. The height profile shows that graphene exhibits a significant out-of-plane height variation $0.6 \pm 0.1 \AA$. The moiré pattern period is extracted to be $15.6 \pm 0.4 \mathrm{~nm}$, which is larger than the value of $14 \mathrm{~nm}$ derived from the $1.8 \%$ lattice mismatch between graphene and h-BN (Fig. 1c). This suggests that the graphene is stretched by $\approx 0.2 \%$ by the h-BN substrate, and the reduced lattice match leads to an expanded moiré pattern. Figure 1d shows ARPES data measured along the $\Gamma-\mathrm{K}$ direction. Dispersions from the $\pi$ and $\sigma$ bands of both graphene and h-BN are observed, and they overlap in most regions, except the $\pi$ band near the $\mathrm{K}$ point. Here, characteristic linear dispersion from the Dirac cone of graphene is observed, while the top of the $\pi$ band from h-BN lies at $-2.2 \mathrm{eV}$, in agreement with the large bandgap of h-BN. The absence of graphene $\pi$ band splitting confirms that the sample is dominated by monolayer graphene. An expected result of the moire potential is the appearance of replicas of the graphene Dirac cone at the same energy but translated by $\boldsymbol{G}_{\mathrm{s}}$ from the graphene $\mathrm{K}$ point (first-generation Dirac cones, see Fig. 1e). The duplicated Dirac cones are obvious in both the Fermi surface map (Fig. 1f) and the dispersion images through the nearest SBZ centres (Fig. 1g-i). The average separation between the original Dirac band and its replicas is $0.044 \pm 0.003 \AA^{-1}$, which is consistent with the observation of the expanded moiré pattern.

We focus on data taken near the SBZ to search for signatures of SDCs away from $E_{\mathrm{F}}$. Figure 2a1-a5 displays intensity maps taken at constant energies between -200 to $-400 \mathrm{meV}$. Corresponding curvature plots $^{26}$ are used to highlight dispersive bands in ARPES data (Fig. 2b1-b5). At $-250 \mathrm{meV}$, a small pocket is visible at the upper-right and lower-right corners of the SBZ (red dots, labelled as $\kappa$ in Fig. 2a1), and is especially clear at $-350 \mathrm{meV}$ and $-400 \mathrm{meV}$. The size of the pockets increases with decreasing energy, which is consistent with conical dispersions. Dispersion images (Fig. 2d1-d5) taken near these corners further support the existence of conical dispersions. When approaching the second-generation Dirac points (SDPs) at $\kappa$ (cuts $\mathrm{d} 1, \mathrm{~d} 2$ and $\mathrm{d} 3$ ), the dispersion relations exhibit a rounded $\mathrm{M}$-shape, with the top of these bands moving towards higher energies-reaching $-0.21 \mathrm{eV}$ at the SDPs (cut d3). After passing the SDPs, the tops of the valence bands move to lower energies (cuts d3, d4 and d5), again in agreement with conical dispersion. We note that around the right corner of the SBZ (labelled as $\kappa^{\prime}$ ), no conical dispersion is observed, suggesting that the two superlattice valleys $\kappa$ and $\kappa^{\prime}$ are inequivalent. Therefore, both the constant-energy maps and the dispersion images presented above reveal directly that SDCs exist at the SBZ corners and only at one of the two superlattice valleys ${ }^{23}$, which is in agreement with the Landau level degeneracy implied from previous quantum Hall effect measurements ${ }^{5,19}$. Such direct information is critical in determining the parameters used to describe the generic band structure of $\mathrm{SDCs}^{10,20}$, and in further understanding other experimental results which probe the electronic structure indirectly.

To better resolve the detailed dispersions of the SDCs, in Fig. 3a we show ARPES data taken near the $\kappa$ point of the SBZ. The conduction bands and valence bands show rounded $\mathrm{W}$ - and $\mathrm{M}$-shapes, respectively, with a large energy separation and a suppression of intensity between them. This indicates the gap opening at the SDCs. The corresponding energy distribution curves (EDCs) are shown in Fig. 3d. In addition to peaks from the conduction and valence bands, there are also non-dispersive peaks (marked by blue ticks) at the band extrema, suggesting that there is significant impurity scattering at the gap edges. From the extracted dispersions and the peak separation at the SDC (Fig. 3g), the gap at the SDCs is approximately $100 \mathrm{meV}$. Direct observation of gapped SDCs around the left corner of the SBZ (equivalent to the two $\kappa$ points discussed above) in the constant-energy maps is difficult, since the intensity in the vicinity of this momentum region is dominated by the original graphene Dirac cone. However, the intensity suppression in EDCs (Fig. 3e,f) from data taken along two high-symmetry directions $\Gamma-\mathrm{K}$ and $\Gamma-\mathrm{M}$ (Fig. 3b,c) prove its existence with a similar gap (Fig. 3h,i). The observation of gapped SDCs at only one of the superlattice valleys $\kappa$ suggests that the inversion-asymmetric perturbation potential from the $\mathrm{h}-\mathrm{BN}$ substrate plays an important role in the electronic structure of graphene/h-BN.

To check whether there is a gap at the original Dirac cone, we dope the graphene/h-BN sample by depositing rubidium to shift the Dirac point below $E_{\mathrm{F}}$ so that it can be measured by ARPES. By shifting the Dirac point to $-0.2 \mathrm{eV}$, the conduction band becomes detectable. Figure 4a1-a5 shows several cuts around the $\mathrm{K}$ point of graphene (see schematic drawing in $(\mathrm{g})$ ). Because photoemitted electrons from higher binding energy have a lower kinetic energy and a smaller in-plane momentum component, the cutting planes are slightly tilted. The extracted dispersions from momentum distribution curves (MDCs, b1-b5) and EDCs (c1-c5) are overplotted as blue dotted and black solid curves in Fig. 4a1-a5. Figure $4 \mathrm{~h}$ shows a zoom-in of the dispersions near the Dirac point. The conduction and valence bands obviously do not touch each 


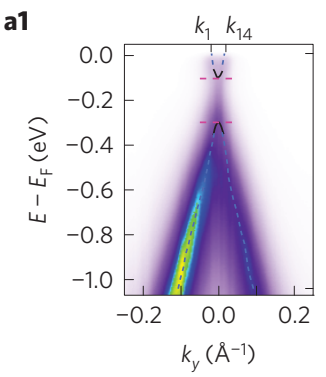

b1

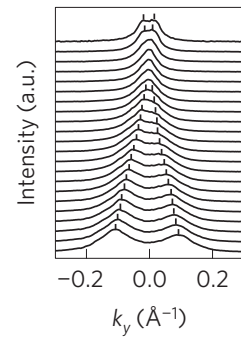

c1

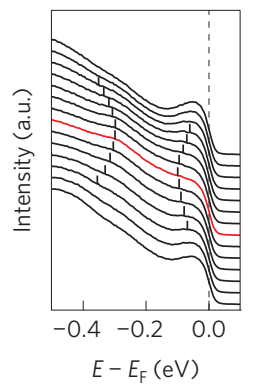

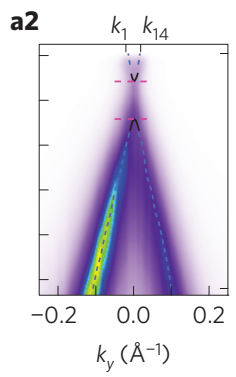

b2

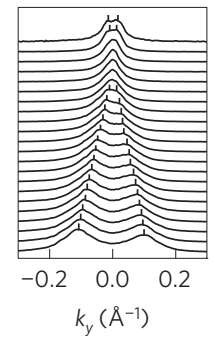

c2

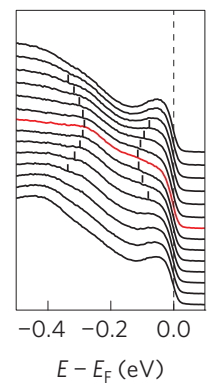

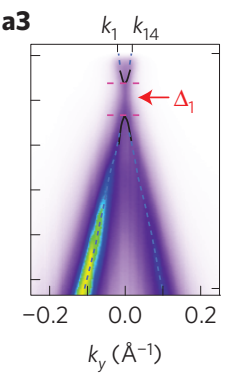

b3

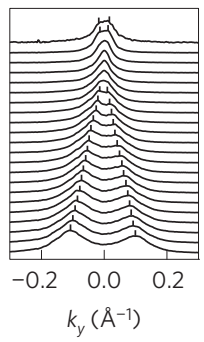

c3

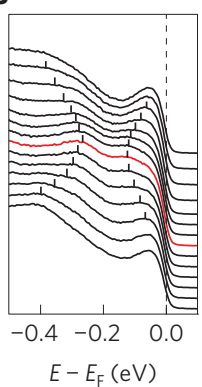

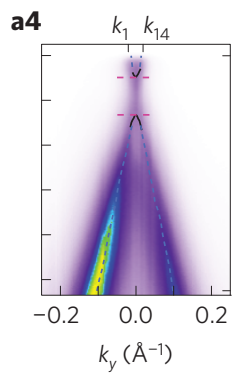

b4

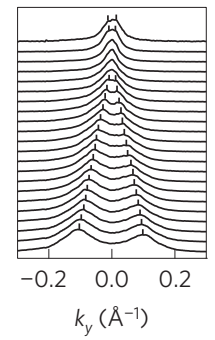

c4

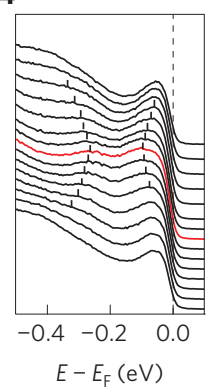

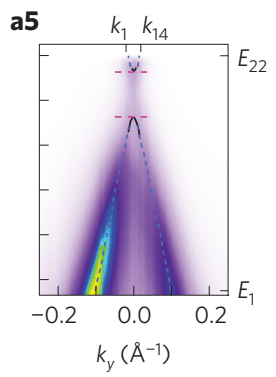

b5

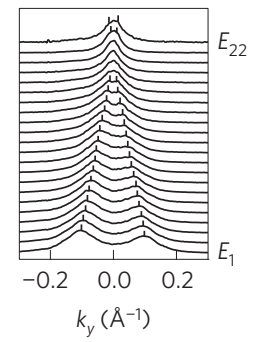

c5

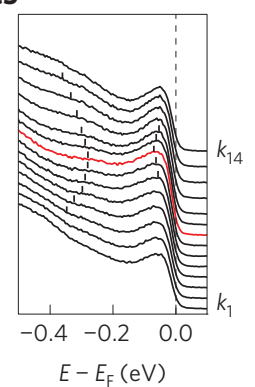

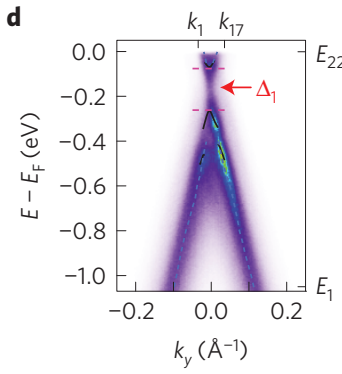

e
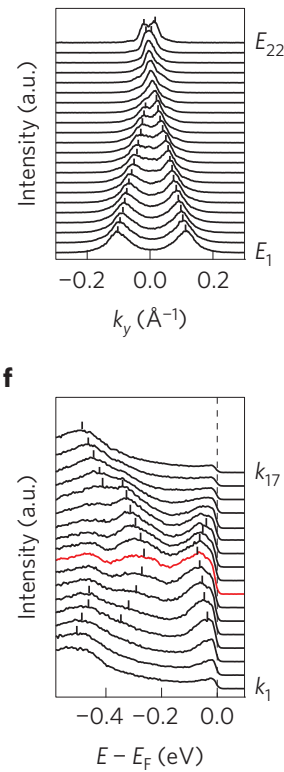
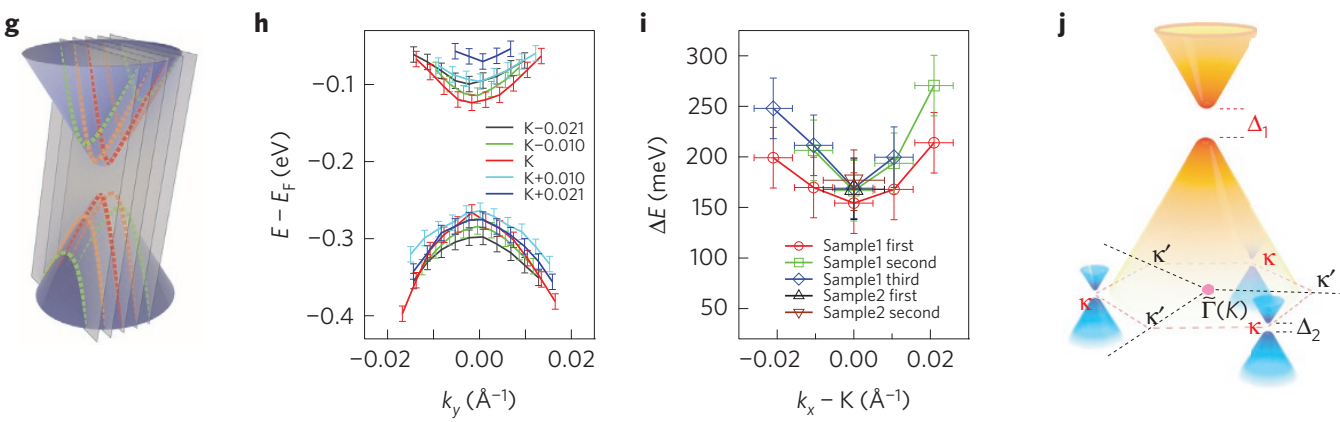

Figure 4 | Gap at the original Dirac point. a1-a5, Band dispersions around the K point from sample 1 after the first doping. The cuts are measured at $k_{x}-K=-0.021,-0.01,0,+0.01$ and $+0.021 \AA^{-1}$, respectively. Blue dotted and black lines indicate dispersions extracted from MDCs and EDCs, respectively. The pink broken lines indicate the bottom of the conduction band and the top of the valence band. b1-b5, Corresponding MDCs for a1-a5,

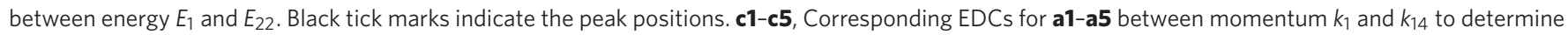

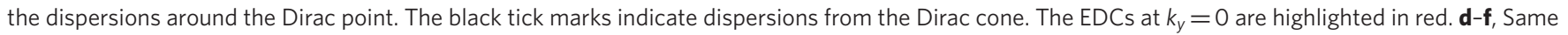
as a3-c3, but from sample 2 after a second doping. $\mathbf{g}$, Schematic drawing of the geometry for cuts a1-a5. h, Zoom-in of the dispersions extracted from c1-c5 to show the evolution of band dispersions when crossing the K point. The error bar is defined as the upper limit when the energy position is clearly offset from the peak position. $\mathbf{i}$, Summary of the energy separation between the bottom of conduction band and the top of valence band for each cut measured on samples 1 and 2 at all doping levels. The minimum separation at the K point gives the gap size. The energy error bar is defined as the sum of

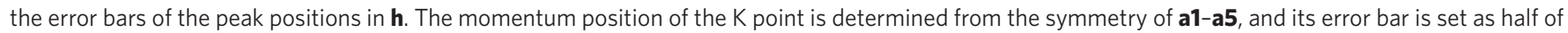
the momentum step size. j, Schematic drawing of the band structure in the graphene/h-BN heterostructure, showing the gapped original Dirac point with gapped SDPs at three out of six corners of the SBZ ( $\kappa$ points).

other when the cutting plane crosses the K point. Such behaviour is an unambigous signature of an excitation gap. Figure $4 \mathrm{~d}-\mathrm{f}$ shows the data through the $\mathrm{K}$ point from sample 2. The dispersion is consistent with sample 1 , while the intensity suppression from the gap at the SDCs is still clearly visible, probably because of the higher sample quality. Figure 4 i summarizes the energy separation between the conduction and valence bands. From the minimum separation at the $\mathrm{K}$ point, the gap is extracted to be $160 \pm 30 \mathrm{meV}$. The raw data for multiple samples at all doping levels can be found in the Supplementary Information.

The extracted gap size at the original Dirac cone from ARPES measurements is much larger than previous measurements, for example, $15-30 \mathrm{meV}$ from transport measurements in transferred graphene/h- $\mathrm{BN}^{4,16}$ and $\sim 40 \mathrm{meV}$ from magneto-optical 
spectroscopy on similar R-PECVD samples ${ }^{24}$, as well as theoretical predictions. Here we discuss a few reasons and the implications. First, since the gap is extracted from the band edges in ARPES and from the density of states in other measurements, gaps probed by other techniques can be much smaller than by ARPES since the density of states rarely drops to zero abruptly at the band edges. Impurities can also contribute to in-gap states and lead to a smaller gap size. In bilayer graphene, it has been suggested that the gap extracted from transport or STM measurements can be underestimated due to additional conductive channels created by defects and charge impurities ${ }^{27}$, and this may also affect the gap size measured in graphene/h-BN. Second, although the maximum gap at the equilibrium layer separation for a perfectly lattice matched heterostructure is predicted to be approximately $50 \mathrm{meV}$ (ref. 13), the gap size increases sharply upon reducing the separation between the graphene and $\mathrm{h}-\mathrm{BN}$ layers ${ }^{13}$. A bandgap opening at the graphene Dirac cone and SDCs can be induced by an inversion-asymmetric mass term in the perturbation potential ${ }^{10,21,23}$, and the mass term can vary by orders of magnitude depending on the assumptions used $^{13,28,29}$. Shortening of the layer separation between graphene and h-BN by $0.2 \AA$ around the equilibrium distance can result in an almost threefold increase of the local mass term ${ }^{13}$. Third, the substantial out-of-plane height variation of $0.6 \AA$ and $0.2 \%$ in-plane strain revealed by atomic force microscopy (AFM) measurements suggest sufficiently large variations in the local mass term and can produce a large average gap ${ }^{13,25}$. Therefore, the large gap size suggests that our epitaxially grown graphene/h-BN samples have much stronger short-range interlayer interaction than the weak van der Waals interaction as commonly believed in ideally flat structures. Although a definitive explanation of the gap opening still requires more theoretical and experimental investigations, our work reports the intriguing electronic structure in a model van der Waals heterostructure, and highlights the important role of the inversionsymmetry-breaking perturbation potential and interfacial atomic structure in the physics of graphene/h-BN heterostructures.

\section{Methods}

Methods, including statements of data availability and any associated accession codes and references, are available in the online version of this paper.

Received 2 January 2016; accepted 12 July 2016; published online 22 August 2016; corrected online 7 October 2016

\section{References}

1. Geim, A. K. \& Grigorieva, I. V. Van der Waals heterostructures. Nature 499, 419-425 (2013).

2. Ponomarenko, L. A. et al. Cloning of Dirac fermions in graphene superlattices. Nature 497, 594-597 (2013).

3. Dean, C. R. et al. Hofstadter's butterfly and the fractal quantum Hall effect in moiré superlattices. Nature 497, 598-602 (2013).

4. Hunt, B. et al. Massive Dirac fermions and Hofstadter butterfly in a van der Waals heterostructure. Science 340, 1427-1430 (2013).

5. Yu, G. L. et al. Hierarchy of Hofstadter states and replica quantum Hall ferromagnetism in graphene superlattices. Nat. Phys. 10, 525-529 (2014).

6. Dean, C. R. et al. Boron nitride substrates for high-quality graphene electronics. Nat. Nano. 5, 722-726 (2010).

7. Xue, J. et al. Scanning tunnelling microscopy and spectroscopy of ultra-flat graphene on hexagonal boron nitride. Nat. Mater. 10, 282-285 (2011).

8. Park, C.-H., Yang, L., Son, Y.-W., Cohen, M. L. \& Louie, S. G. Anisotropic behaviours of massless Dirac fermions in graphene under periodic potentials. Nat. Phys. 4, 213-217 (2008).

9. Park, C.-H., Yang, L., Son, Y.-W., Cohen, M. L. \& Louie, S. G. New generation of massless Dirac fermions in graphene under external periodic potentials. Phys. Rev. Lett. 101, 126804 (2008).

10. Wallbank, J. R., Patel, A. A., Mucha-Kruczyński, M., Geim, A. K. \& Fal'ko, V. I. Generic miniband structure of graphene on a hexagonal substrate. Phys. Rev. $B$ 87, 245408 (2013).
11. Ortix, C., Yang, L. \& van denBrink, J. Graphene on incommensurate substrates: trigonal warping and emerging Dirac cone replicas with halved group velocity. Phys. Rev. B 86, 081405 (2013).

12. San-Jose, P., Gutiérrez-Rubio, A., Sturla, M. \& Guinea, F. Spontaneous strains and gap in graphene on boron nitride. Phys. Rev. B 90, 075428 (2014).

13. Giovannetti, G., Khomyakov, P. A., Brocks, G., Kelly, P. J. \& van den Brink, J. Substrate-induced band gap in graphene on hexagonal boron nitride: $a b$ initio density functional calculations. Phys. Rev. B 76, 073103 (2007).

14. Amet, F., Williams, J. R., Watanabe, K., Taniguchi, T. \& Goldhaber-Gordon, D. Insulating behavior at the neutrality point in single-layer graphene. Phys. Rev. Lett. 110, 216601 (2013).

15. Gorbachev, R. V. et al. Detecting topological currents in graphene superlattices. Science 346, 448-451 (2014).

16. Woods, C. R. et al. Commensurate-incommensurate transition in graphene on hexagonal boron nitride. Nat. Phys. 10, 451-456 (2014).

17. Shi, Z. et al. Gate-dependent pseudospin mixing in graphene/boron nitride moiré superlattices. Nat. Phys. 10, 743-747 (2014).

18. Yankowitz, M. et al. Emergence of superlattice Dirac points in graphene on hexagonal boron nitride. Nat. Phys. 8, 382-386 (2012).

19. Yang, W. et al. Epitaxial growth of single-domain graphene on hexagonal boron nitride. Nat. Mater. 12, 792-797 (2013).

20. Jung, J., Raoux, A., Qiao, Z. \& MacDonald, A. H. Ab initio theory of moiré superlattice bands in layered two-dimensional materials. Phys. Rev. B 89, 205414 (2014).

21. Moon, P. \& Koshino, M. Electronic properties of graphene/hexagonal-boron-nitride moiré superlattice. Phys. Rev. B 90, 155406 (2014)

22. van Wijk, M. M., Schuring, A., Katsnelson, M. I. \& Fasolino, A. Moiré patterns as a probe of interplanar interactions for graphene on h-BN. Phys. Rev. Lett. 113, 135504 (2014).

23. Jung, J., DaSilva, A. M., MacDonald, A. H. \& Adam, S. Origin of band gaps in graphene on hexagonal boron nitride. Nat. Commun. 6, 6308 (2015).

24. Chen, Z.-G. et al. Observation of an intrinsic bandgap and Landau level renormalization in graphene/boron-nitride heterostructures. Nat. Commun. 5, 4461 (2014).

25. Bokdam, M., Amlaki, T., Brocks, G. \& Kelly, P. J. Band gaps in incommensurable graphene on hexagonal boron nitride. Phys. Rev. B 89, 201404 (2014).

26. Zhang, P. et al. A precise method for visualizing dispersive features in image plots. Rev. Sci. Instrum. 82, 043712 (2011).

27. Rutter, G. M. et al. Microscopic polarization in bilayer graphene. Nat. Phys. 7, 649-655 (2011).

28. Kindermann, M., Uchoa, B. \& Miller, D. L. Zero-energy modes and gate-tunable gap in graphene on hexagonal boron nitride. Phys. Rev. B 86, 115415 (2012)

29. Song, J. C. W., Shytov, A. V. \& Levitov, L. S. Electron interactions and gap opening in graphene superlattices. Phys. Rev. Lett. 111, 266801 (2013).

30. Slawinska, J., Zasada, I. \& Klusek, Z. Energy gap tuning in graphene on hexagonal boron nitride bilayer system. Phys. Rev. B 81, 155433 (2010).

\section{Acknowledgements}

We thank V. I. Fal'ko for useful discussions. This work is supported by the National Natural Science Foundation of China (Grant No. 11274191, 11334006, and 11427903), Ministry of Science and Technology of China (Grant No. 2015CB921001,

2016YFA0301004) and Tsinghua University Initiative Scientific Research Program (2012Z02285). E.Y.W. is grateful for support from the Advanced Light Source Doctoral Fellowship Program. The Advanced Light Source is supported by the Director, Office of Science, Office of Basic Energy Sciences, of the US Department of Energy under Contract No. DE-AC02-05CH11231.

\section{Author contributions}

S.Z. designed the research project. E.W., S.D., W.Y., M.Y., G.W., K.D., A.V.F. and S.Z. performed the ARPES measurements and analysed the ARPES data. X.L., S.W., G.C., G.Z. and Y.Z. prepared the graphene samples. J.J. discussed the data. E.W. and S.Z. wrote the manuscript, and all authors commented on the manuscript.

\section{Additional information}

Supplementary information is available in the online version of the paper. Reprints and permissions information is available online at www.nature.com/reprints.

Correspondence and requests for materials should be addressed to S.Z.

\section{Competing financial interests}

The authors declare no competing financial interests. 


\section{Methods}

Sample preparation. Graphene samples were directly grown on h-BN substrates by the epitaxial method, as specified in ref. 19. As-grown samples were characterized by tapping mode atomic force microscopy (MultiMode IIId, Veeco Instruments) at room temperature in ambient atmosphere. We used freshly cleaved mica as shadow masks for metal electrode deposition. Using the micromanipulator mounted on an optical microscope, the freshly cleaved mica flakes were accurately transferred onto the substrates, covering one side of the substrates with a greater area of target graphene/h-BN samples. The contact metal ( $\sim 40 \mathrm{~nm} \mathrm{Pd}$ or Au) was deposited on the non-mica-covered area with a smaller area of target graphene/ h-BN samples. The samples were then annealed at $200^{\circ} \mathrm{C}$, after removing the mica flakes.
ARPES measurement. Measurements were performed using ARPES instruments based at the synchrotron-radiation light source at beamline 12.0.1 of the Advanced Light Source at Lawrence Berkeley National Laboratory (LBNL). The data were recorded by a Scienta R3000 at BL 12.0.1 with photon energies of 50 and $60 \mathrm{eV}$. The energy and angle resolution are better than $30 \mathrm{meV}$ and $0.2^{\circ}$, respectively. Before measurements, the samples were annealed at $200^{\circ} \mathrm{C}$ for $2-3 \mathrm{~h}$ until clean surfaces were exposed. All measurements were performed between $10-20 \mathrm{~K}$ and under a vacuum better than $5 \times 10^{-11}$ torr. The rubidium deposition was achieved by heating an SAES commercial dispenser in situ.

Data availability. The data that support the plots within this paper and other findings of this study are available from the corresponding author upon reasonable request. 